Document downloaded from:

http://hdl.handle.net/10251/61353

This paper must be cited as:

Vilar Mateo, R.; Martí Sendra, J.; Alain Le Fevre; Bosshard, O.; Magne, F.; Lefevre, A. (2013). Point to Multipoint Backhaul Architecture for 3G/4G Networks and Small Cell Deployment. En Antennas and Propagation in Wireless Communications (APWC), 2013 IEEE-APS Topical Conference on. Institute of Electrical and Electronics Engineers (IEEE). 1-4. doi:10.1109/APWC.2013.6624942.

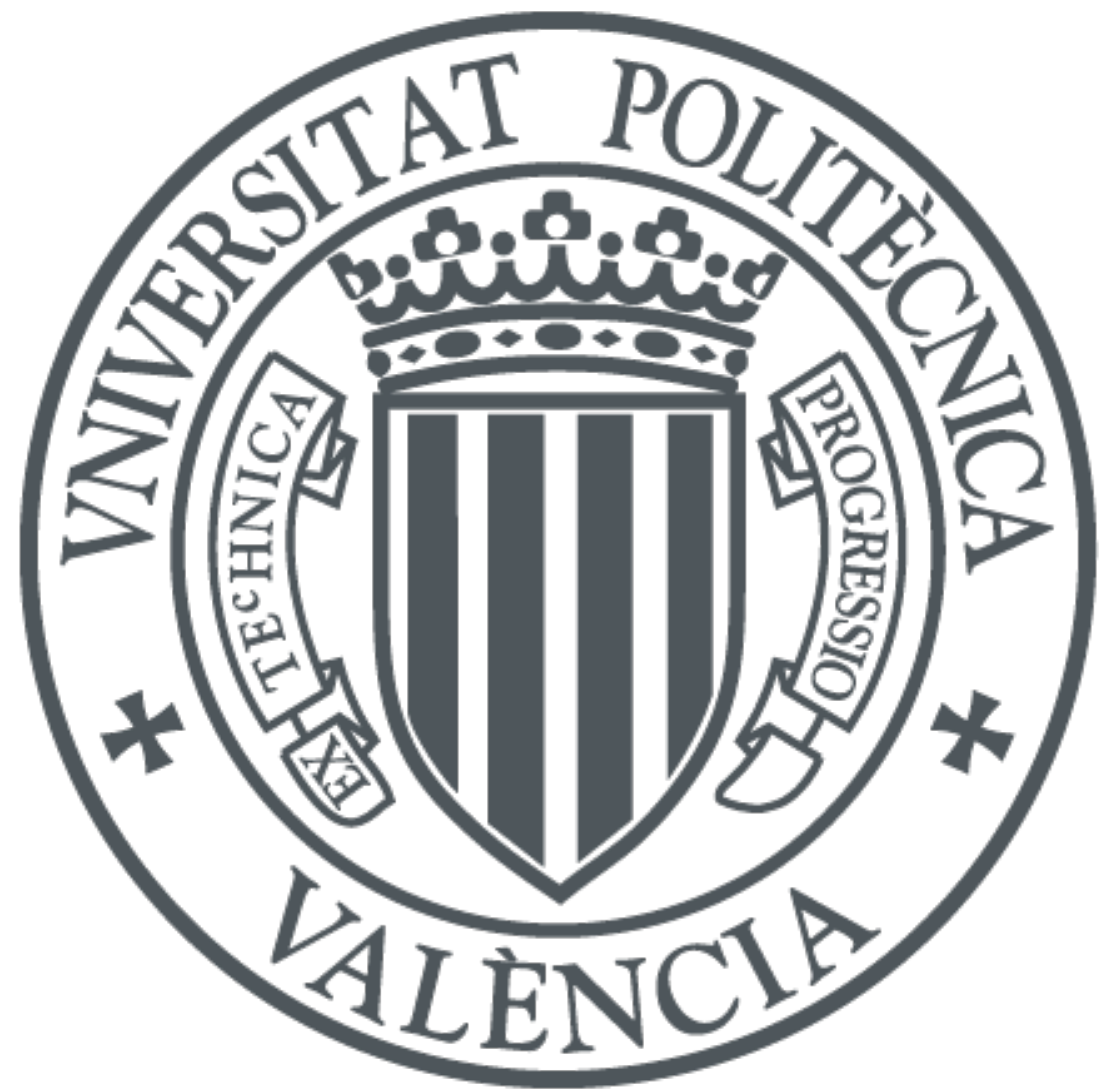

The final publication is available at

http://dx.doi.org/10.1109/APWC.2013.6624942

Copyright Institute of Electrical and Electronics Engineers (IEEE)

Additional Information 


\title{
Point to Multipoint Backhaul Architecture for 3G/4G Networks and Small Cell Deployment
}

\author{
R. Vilar ${ }^{1}$, J. Marti ${ }^{1}$,O. Bosshard ${ }^{2}$, F. Magne ${ }^{2}$, A. LeFevre ${ }^{3}$
}

\begin{abstract}
Demand for mobile broadband services is continually increasing, requiring operators to provide more and more capacity from their radio access networks. LTE and small cells can offer a promising solution to provide almost unlimited coverage and capacity. However, backhaul technologies can be expensive in terms of both CAPEX and OPEX, and none of the traditional solutions provides the necessary combination of capacity and cost-efficiency. This paper defines a Q-band point to multipoint backhaul architecture that provides multi-gigabit capacity in a costeffective manner. Apart from the network architecture, the key nodes comprising the network will be described.
\end{abstract}

\section{INTRODUCTION}

Today's proliferation of smartphones and tablets running applications that make an extensive use of data traffic, such as multi-player games or video streaming on-the-go, has in turn resulted in a colossal increase in bandwidth demand on the $3 \mathrm{G}+/ 4 \mathrm{G}$ networks. Actually, CISCO's top line global mobile data traffic growth projects show mobile data traffic increasing 26x fold from 2010 to 2015 [1]. Looking at these figures, mobile network operators (MNOs) are experiencing difficulties in meeting these traffic demands and need to find ways to overcome this problem in a cost-effective manner. At the first stage, the evolution to $3 \mathrm{G}$ and $4 \mathrm{G} / \mathrm{LTE}$ mobile technologies provides a path to more efficient use of radio spectrum and progressively higher uplink and downlink speeds to each user. However, operators' forecasts show that additional steps are required to provide the required amount of bandwidth. As a result, operators have begun to introduce small cells into their networks since this solution has recently emerged as a more cost-effective way for MNOs to improve the coverage and capacity of their mobile services. However, to leverage the benefits of small cells, there are some challenges to overcome. One of the most significant issues is how to provide scalable, flexible mobile backhaul to connect small cells back into the network, without breaking the small cell business case.

A further issue is that the traditional backhaul solutions based on fiber, copper, and microwaves can no longer keep up [2]. Fiber provides a very high capacity and low-latency connection; however it is neither fast to install nor cheap to do so since fiber is not a cost-effective way of connecting up a continuously evolving set of small cells. Digital subscriber line (DSL) is more widely deployed than fiber today and, where already installed, it can provide a basic level of connectivity; however, it will not provide the peaks needed for a good end user quality of experience. Non-line of sight (NLoS) wireless solutions would offer a good solution were it not for the small cells and Wi-Fi hotspots already using the entire low frequency spectrum available. On the other hand, point to point (PTP) microwave radio links are a well-established and mature technology that has been often applied to backhaul mobile traffic. However, traditional PTP architectures require large antenna dishes at each end of the link, resulting in innately equipment-heavy and expensive total cost of ownership (TCO) network. In a point to multipoint (PMP) solution, the central base station serves simpler and less costly subscriber units which are placed at the end points. As a consequence, a PMP architecture can be a more cost-effective solution for operators looking to deliver high capacity data services at a low TCO. However, although microwave-based PMP solutions improve the CAPEX/OPEX factors, this technology is not suitable for providing the required bandwidth demand of next generation wireless networks. Therefore, the key challenge is how to deploy backhaul LTE small cells in an affordable way, without sacrificing capacity.

This paper defines the architecture for future mobile backhaul and access wireless networks as proposed in the framework of the SARABAND project. More concretely, the SARABAND project is proposing an architecture providing multi-gigabit capacity in a cost-effective manner by combining point to multipoint transmissions and millimeter wave (mm-wave) technology in the Q-band. Apart from the network definition, the key type of nodes comprising the SARABAND architecture (transmission hub and network terminal equipment) will be described.

\section{MILLIMETRE WAVE POINT TO MULTIPOINT SOLUTION}

Point to multipoint systems are inherently characterized by the ability for a single radio or hub terminal to sustain links with multiple remote terminals. This ability to support $n$ radio links with only $(n+1)$ radios is PMP's fundamental advantage over PTP systems, which need to bear the higher cost of $2 n$ radios to support the same $n$ links. This

\footnotetext{
${ }^{1}$ Valencia Nanophotonics Technology Center, Universitat Politècnica de València, Camino de Vera s/n, 46022Valencia, Spain, e-mail: rutvima@ntc.upv.es, tel.: +34 963879746 , fax: +34 963877827.

${ }^{2}$ Bluwan UK Ltd, Berkeley Square, London, W1J 6BR, United Kingdom, e-mail: sales@bluwan.com, tel.: +44 (0) 203 384 9810 , fax: +44 (0) 2033849811 .

${ }^{3}$ Thales Communications \& Security, Avenue des Louvresses, 92622 Gennevilliers Cedex, France, e-mail: alain.lefevre@thalesgroup.com, tel.: +330141303418, fax: +330141303418
} 
advantage means a significant decrease in CAPEX. Additionally, as a consequence of not requiring dedicated $n$ links to serve $n$ endpoints, a PMP architecture can efficiently manage scarce spectrum resources as it can dynamically allocate capacity amongst $n$ endpoints. This provides for a flexible network topology which lends itself to subscriber adds, moves and changes thus lowering OPEX for an operator and facilitating rapid subscriber acquisition.

However, although traditional PMP backhaul, such as the ones operating in the 10 or $28 \mathrm{GHz}$ bands, improves resources utilization, microwave technology does not provide the required capacity to meet future market needs in terms of bandwidth.

Mm-wave based technologies are well positioned to serve the needs of these applications into the foreseeable future. Solutions based on lower frequency microwave wireless systems may meet the short term bandwidth demand; however, when the cost of such solutions and the cost of spectrum licenses are factored in, mm-wave solutions begin to appear more attractive compared to other options such as laying fiber to a cell tower. Therefore, mmwave technology, especially in the Q-band (40,5 43,5 GHz), fits nicely into the new backhaul scenarios and fixed service enhancement needs while providing:

- Large amount of spectral bandwidth available suitable for wide channels ( $3 \mathrm{GHz}$ bandwidth, 6 $\mathrm{GHz}$ with cross-polarization).

- Adaptive throughput rate, which allows efficient and flexible deployment.

- Flexible network topology support.

- Compact and light equipment.

- Simple and cost-effective last mile distribution from fiber optics points.

- Low radiation levels.

Moreover, Q-band links can withstand high amounts of interference and weather conditions such as rain, fog, and snow. In comparison, signals in the 57-64 GHz spectrum (U-band) are subject to the resonance of oxygen molecules and hence propagation in this spectrum is severely attenuated, leading to very short link ranges. The 70-80 GHz spectrum (E-band) is dedicated to PTP microwave links, and integration of MMICs (Monolithic Microwave Integrated Circuit) modules is not yet possible at a low cost.

Therefore, mm-wave PMP solution has been proposed in SARABAND project as a promising solution that can backhaul LTE small cells in an affordable way while fulfilling capacity requirements.

\section{SARABAND NETWORK ARCHITECTURE \& NETWORK NODES}

\subsection{Network architecture}

SARABAND architecture is a hierarchical, PMP Ethernet-based network composed of nodes linked by radio transmissions, and remotely managed by a backhaul NMS instance. The solution is architected as a distribution tree connecting a service provider's point of presence (PoP) to relay nodes an ultimately reaching: subscriber terminals, grouped terminals and mobile base stations.

The architecture uses a $1 \mathrm{GHz}$-wide transmitter that can be loaded with a multiplex of independent channels, aggregating several $100 \mathrm{Mbps}$ half-duplex time division duplex (TDD) channels to provide the required throughput, up to 2 Gbps half-duplex per 1 $\mathrm{GHz}$ radio transmission. Indeed, a base station can be configured to use bandwidths from only $40 \mathrm{MHz}$ up to all $3 \mathrm{GHz}$ available at the Q-band (@40GHz using 3 transmitters fully loaded). The more spectrum that is available the more bandwidth can be delivered on a point to multipoint sector to facilitate $\mathrm{Nx} 100 \mathrm{Mbps}$ services [3].

The network comprises two main types of nodes:

- Transmission Hubs (TH) to carry traffic in the core network, and to connect several terminals or a relay node $(\mathrm{RN})$. The $\mathrm{TH}$ switches the packet traffic between radio thoroughfares to provide connectivity inside the backhaul network.

- Network Terminal Equipments (NTE) to deliver basic Service Interfaces to customer's PoP. They establish a single connectivity to the TH where they are connected through a VLAN for each of them.

These network nodes are composed of different elements such as radio frequency modules, multiplexers, modems and antennas. The radios have to be of low noise figure and high power to support many channels (modem) and extended cell range. The radios have also to be of low phase noise to enable increased modulation and higher spectral efficiency. For increased cell range but also reduced risk of interference and increased frequency re-use, the antennas have to be very directive and of high gain, being smart antennas the optimal solution.

More concretely, the $\mathrm{TH}$ is composed of a transmitter $(\mathrm{Tx})$ and a receiver $(\mathrm{Rx})$ with high-gain or programmable multi-beam antennas. The Tx and the $\mathrm{Rx}$ are connected to a CAI (C-band Air Interface) multiplexer/demultiplexer and this in turn to a stack of $802.11 \mathrm{n}$ standard based modems (from 4 to 20: up to 20 channels of $40 \mathrm{MHz}$ spaced by $15 \mathrm{MHz}$, resulting in $50 \mathrm{MHz}$ spectrum per channel using 802.11 m modulation). 

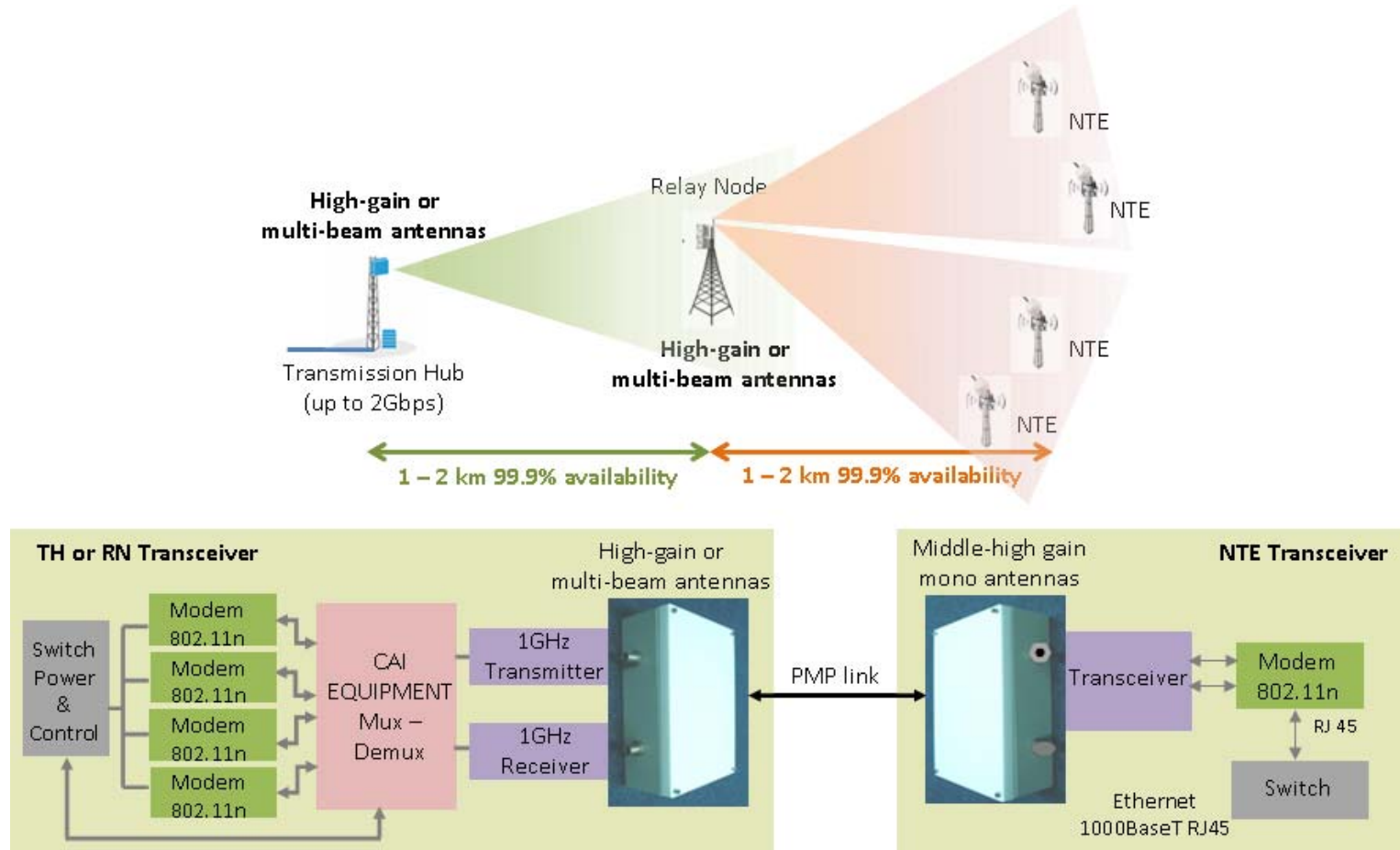

Figure 1. Backhaul network architecture

These modems are then plugged on an Ethernet switch which performs the aggregation (802.3ad). The $\mathrm{RN}$ is also a transmission Hub plus a link "upstream" (NTE to the TH). On the other hand, the NTE comprises a transceiver with directive antennas. The transceiver is connected to a modem 802.11n through a combiner splitter. A detailed picture of the backhaul network is shown in Figure 1.

\subsection{Network nodes}

SARABAND architecture is composed of network nodes with high-gain and multi-beam antennas to enable any configuration of hierarchical point to multipoint structure, and radios with high bandwidth and a high degree of integration to meet performance, cost-efficiency and acceptability. However, with the existing Q-band antennas, Tx's and Rx's, the PMP backhaul in mm-wave is limited either in range or in number of base stations connected. Therefore, to guarantee the segment coverage and the expected capacity and throughput on each segment of the network, some improvements in terms of antennas and radio technologies in Q-band must be addressed. More concretely, links between the central transmission hub and the relay node will require low profile, high gain antennas to enhance throughput whereas links between relay nodes and base stations or terminals will require programmable multi-beam antennas to enhance coverage, reduce interferences and save energy. To specify the antenna parameters required to obtain the desired performance, a complete model of the system TH-NTE including all gains and losses of the sub-assemblies, the propagation model, antenna diagram, channelization isolation, etc. has been developed. This model provides the parameter configuration of the system and delivers its performances as a function of the antennas main values. The results coming from this model point out that TH-RN antennas should have a 36-dBi gain, RN-NTE antennas a gain of $24 \mathrm{dBi}$ and NTE antennas a gain of $30 \mathrm{dBi}$ (Table 1). To obtain the target gains on each network segment, novel antennas designs in Q-band with a small form factor, programmables, and multi-beam capability based on sub-wavelength Lens and Circular Switched Parasitic Array (CSPA) technologies are being developed at this stage of the project.

\begin{tabular}{|l|l|l|c|c|}
\hline Application & Capacity & Range & $\begin{array}{l}\text { Gain } \\
(\mathrm{dBi})\end{array}$ & Dimension \\
\hline Lens Antenna & Gigabits & $\begin{array}{l}1- \\
3 \mathrm{~km}\end{array}$ & 36 & $25 \mathrm{~cm}$ \\
\hline TH-RN & $\begin{array}{l}\mathrm{N} \mathrm{x} \\
120 \mathrm{Mbps}\end{array}$ & $\begin{array}{l}300 \mathrm{~m} \\
-1 \mathrm{~km}\end{array}$ & 24 & $<20 \mathrm{~cm}$ \\
\hline RN-NTE & & 30 & $<20 \mathrm{~cm}$ \\
\hline NTE & & $500 \mathrm{~m}$ & 16 & - \\
\hline CSPA Antenna & $\begin{array}{l}\mathrm{N} x \\
\begin{array}{l}\text { RN (multi- } \\
\text { beam ant.) }\end{array} \\
120 \mathrm{Mbps}\end{array}$ & & & \\
\hline
\end{tabular}

Table 1. Target antenna gains on each network segment

Concerning high-gain antennas, compared to the classical bulk lens antenna approach, the objective here is to obtain a lower profile antenna with a higher 
radiation efficiency. The cost-effective configuration under study consists of a patch-based feeder combined with a diffractive lens. A first prototype is currently being assembled and will be characterized soon.

On the other hand, with respect to multi-beam antennas, two approaches are under study. This first approach is based on multi-beam lens antennas with the objective to provide directive links to individual or grouped subscribers within a limited field of view. This solution uses a passive distributor component that splits a collimated beam into the required number of beams in the desired directions. Preliminary results have proved the concept viability. The second approach is based on CSPA technology with the objective to explore the capability of an agile antenna with a large field of view that adapts dynamically to the changing environment in a capillary network and forms the antenna radiation pattern to provide optimum system performance and high data rate. The antenna needs to have a gain of $16 \mathrm{dBi}$ with a beamwidth of $6^{\circ} 60^{\circ}$ to cover an area of $270^{\circ}$. The design based on a printed patch CSPA seems to be the most promising solution. The center patch element will be the active radiating element and the surrounding elements will be selectively switched to the state of reflectors to direct the beam in the required direction of radiation. A circular flared horn attached to the antenna can provide the required gain and vertical beam width. A first fixedbeam demonstrator of the circular switched parasitic array antenna has been manufactured and is currently subject to intensive measurements.

Concerning Q-band radio frequency modules, 40$44 \mathrm{GHz}$ isolator/circulator, and novel chipsets for upand down-conversion have been developed. Work on circulators was focused on the modification (adaptation) of circulator traces and bonding geometry improvement to reach more than $15 \mathrm{~dB}$ isolation between ports and less than $1,5 \mathrm{~dB}$ loss. The new chipsets for up- and down-conversion improve the gain, achieving the $18-\mathrm{dBm}$ power target for the Up-converter and 33-dB gain for the Down-converter with 2,5-dB noise factor.

\section{CONCLUSION}

This paper presents the Q-band PMP backhaul architecture as proposed in the SARABAND project. This solution provides multi-Gbps capacity in a costeffective manner by exploiting PMP transmissions and the Q-band spectrum. Additionally, this architecture provides huge advantages in CAPEX for access capillarity and in OPEX for backhaul.

\section{Acknowledgments}

The European Commission is gratefully acknowledged for partial funding of the ICT-2011288267 SARABAND project in the 7 th Framework Programme.

\section{References}

[1] Cisco Article: "Cisco forecasts 18-fold increase in mobile data by 2016 ".

[2] J. Robson, L. Hiley, "Easy small cell backhaul: An analysis of small cell backhaul requirements and comparison of solutions", Cambridge Broadband Network Limited, Feb. 2012.

[3] Bluwan, "Key benefits of Bluwan FTTA: A TDD PTMP architecture", trovaconsulting, 2011. 